Article

\title{
Interplay of Enzyme Therapy and Dietary Management of Murine Homocystinuria
}

\author{
Insun Park ${ }^{1}$, Erez M. Bublil ${ }^{2}$, Frank Glavin ${ }^{2}$ and Tomas Majtan ${ }^{1, *}$ \\ 1 Section of Genetics and Metabolism, Department of Pediatrics, University of Colorado Anschutz Medical \\ Campus, Aurora, CO 80045, USA; insun.park@ucdenver.edu \\ 2 Orphan Technologies Ltd., 8640 Rapperswil, Switzerland; erez.b@neopharmgroup.com (E.M.B.); \\ frank.glavin@neovii.com (F.G.) \\ * Correspondence: tomas.majtan@cuanschutz.edu; Tel.: +1-303-724-3813
}

Received: 31 July 2020; Accepted: 18 September 2020; Published: 22 September 2020

\begin{abstract}
Albeit effective, methionine/protein restriction in the management of classical homocystinuria (HCU) is suboptimal and hard to follow. To address unmet need, we developed an enzyme therapy (OT-58), which effectively corrected disease symptoms in various mouse models of HCU in the absence of methionine restriction. Here we evaluated short- and long-term efficacy of OT-58 on the background of current dietary management of HCU. Methionine restriction resulted in the lowering of total homocysteine (tHcy) by 38-63\% directly proportional to a decreased methionine intake (50-12.5\% of normal). Supplemental betaine resulted in additional lowering of tHcy. OT-58 successfully competed with betaine and normalized tHcy on the background of reduced methionine intake, while substantially lowering tHcy in mice on normal methionine intake. Betaine was less effective in lowering tHcy on the background of normal or increased methionine intake, while exacerbating hypermethioninemia. OT-58 markedly reduced both hyperhomocysteinemia and hypermethioninemia caused by the diets and betaine in HCU mice. Withdrawal of betaine did not affect improved metabolic balance, which was established and solely maintained by OT-58 during periods of fluctuating dietary methionine intake. Taken together, OT-58 may represent novel, highly effective enzyme therapy for HCU performing optimally in the presence or absence of dietary management of HCU.
\end{abstract}

Keywords: inborn error of metabolism; rare disease; tandem mass spectrometry; mouse model; enzyme replacement therapy; sulfur metabolism; homocysteine

\section{Introduction}

Classical homocystinuria (HCU; OMIM\# 236200) is a rare inborn error of sulfur amino acid metabolism caused by substantially reduced or entirely missing cystathionine beta-synthase (CBS) activity [1]. Human CBS is a complex enzyme with intricate regulation responsible for the condensation of homocysteine (Hcy), a toxic intermediate of methionine (Met) metabolism, with serine to form cystathionine (Cth), thus directing the flux of organic sulfur irreversibly toward the biosynthesis of cysteine (Cys) [2] (Figure 1). Consequently, a lack of CBS activity leads to extremely elevated plasma and tissue Hcy concentrations, which represent a biochemical hallmark of HCU. The clinical consequences include skeletal and connective tissue defects, impaired vision, mental retardation, and vascular complications, particularly stroke and deep vein thrombosis, which often lead to the premature death of HCU patients [1]. The major objective of treatment is to reduce the accumulation of Hcy and maintain it as close as possible to normal levels (5-15 $\mu \mathrm{M}$; Figure 1). Recent guidelines for the management of HCU recommend maintaining plasma total Hcy (tHcy) below the $100 \mu \mathrm{M}$ threshold, which, rather than being an ideal target for Hcy levels, represents a high but achievable target that may help in reducing some of the clinical complications [3]. Interestingly, a $50 \mu \mathrm{M}$ tHcy 
threshold was recommended for the less affected individuals, so-called pyridoxine responders, in whom residual CBS activity can be stimulated by supplemental pyridoxine (vitamin $\mathrm{B}_{6}$ ). Its active form pyridoxal-5' -phosphate represents a catalytic cofactor of CBS. Different plasma tHcy thresholds for the two groups of patients affected by HCU and buildup of Hcy testify that these levels were set not as desired or ideal but rather as achievable targets that are attainable by the current standard therapy, thus further emphasizing unmet need. In addition, the majority of patients respond only partially to pyridoxine or not at all. The metabolic balance of HCU patients is thus controlled by dietary protein/Met restriction to decrease flux in the organic sulfur metabolism. In addition, betaine (Bet) supplementation was found beneficial to patients struggling with their diet to achieve therapeutic goals. Although dietary management could be quite effective, the poor taste of Met-free formula or Bet and the fishy odor resulting from Bet administration lead to poor compliance and subsequent exacerbation of clinical complications $[1,3,4]$.

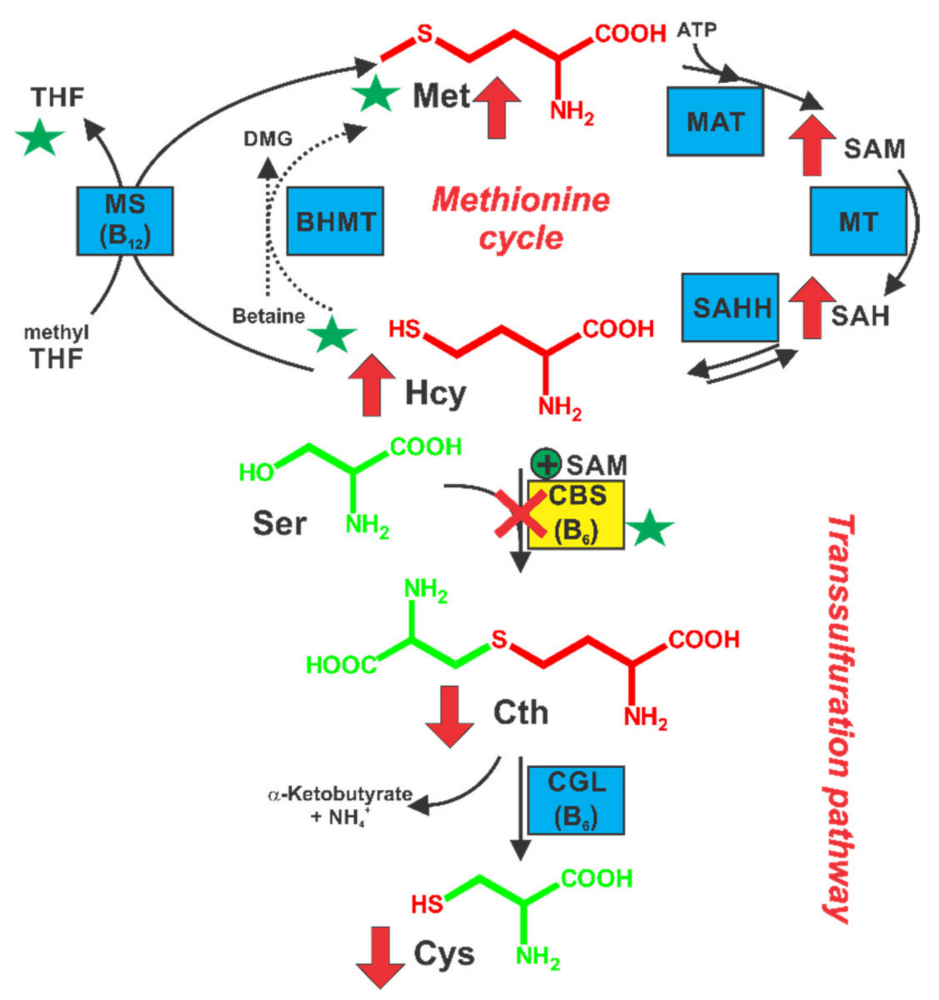

Figure 1. Sulfur amino acid metabolism and management of homocystinuria (HCU). In HCU, sulfur amino acid metabolism is disrupted due to deficient cystathionine beta-synthase (CBS) activity (red cross), which leads to the elevation of upstream metabolites homocysteine (Hcy), methionine (Met), S-adenosylmethionine (SAM), and S-adenosylhomocysteine (SAH) and decrease of downstream metabolites cystathionine (Cth) and cysteine (Cys) as indicated by red arrows. Green stars designate locations, where the current treatments for HCU work: restriction of dietary Met and protein intake to decrease production of Hcy, pyridoxine supplementation to stimulate residual CBS activity and folate/betaine supplementation to promote Hcy re-methylation back to Met. cystathionine $\gamma$-lyase (CGL); di-MethylGlyoxime (DMG); S-adenosylhomocysteine hydrolase (SAHH); Bet-Hcy-methyltransferase (BHMT).

To address unmet need, we developed OT-58, a novel enzyme therapy for HCU [5-8]. OT-58 is a recombinant, truncated, human CBS C15S variant modified with $20 \mathrm{kDa}$ linear N-hydroxysuccinimide ester-activated polyethylene glycol chains. When administered subcutaneously to various mouse models of HCU, OT-58 caused substantial reduction of tHcy and correction of metabolic balance. More importantly, the improved biochemical profile resulted in the correction of survival, liver disease [6], facial alopecia, ciliary zonules [7], endothelial dysfunction, cognitive impairment, body composition, 
and bone mineralization in HCU mice [8]. Notably, most of these results were accomplished in HCU mice maintained on unrestricted Met intake in the form of standard rodent chow with no additional supplements. However, for real-world scenarios of protein/Met restriction, Bet supplementation, and poor compliance with these dietary measures, it is important to understand how OT-58 performs under the challenge of current mainstay therapies, which reduce the availability of Hcy substrate (Met restriction) or directly compete for it (Bet supplementation). Therefore, we evaluated the shortand long-term efficacy of OT-58 to correct the metabolic profile of HCU mice on the background of graded Met restriction, Cys or Bet supplementation, withdrawal of Bet, and fluctuations in Met intake to model current dietary management strategies treating HCU and poor adherence of patients to the dietary management of HCU.

\section{Materials and Methods}

\subsection{Test Compounds}

OT-58, a human, truncated CBS carrying C15S mutation covalently modified with a linear 20 kDa N-hydroxysuccinimide ester polyethylene glycol (ME-200GS; NOF), was prepared as described elsewhere [5]. The OT-58 batch used in the study was 1618TR1 with a protein concentration of $22 \mathrm{mg} / \mathrm{mL}$ and average CBS specific activity of $1332 \pm 45 \mathrm{U} / \mathrm{mg}$ of protein.

\subsection{Diets}

Prior to the study execution, animals were maintained on a standard complete extruded diet 2920X (Envigo). Amino acid-defined diets (Envigo) varying in content of sulfur amino acids Met or Cys were used in the below described animal studies. Their compositions and nutritional information are summarized in Table 1. Briefly, the diet designated MET4.0 containing $4.0 \mathrm{~g} / \mathrm{kg}$ Met and $3.5 \mathrm{~g} / \mathrm{kg}$ Cys (as cystine) had similar content of sulfur amino acids as the standard complete diet 2920X (5 g/kg Met and $3 \mathrm{~g} / \mathrm{kg}$ Cys) and thus served as a normal control diet. Diets designated MET2.0, MET1.0, and MET 0.5 had reduced contents of Met compared to MET4.0 to 2.0, 1.0, and $0.5 \mathrm{~g} / \mathrm{kg}$, respectively. Similarly, the diet designated as MET8.2 had roughly doubled the Met content $(8.2 \mathrm{~g} / \mathrm{kg})$ compared to MET4.0. The content of Cys in all the MET diets was identical (i.e., $3.5 \mathrm{~g} / \mathrm{kg}$ ). In contrast, the diet designated as $3 x$ CYS had Met content same as the MET4.0, but its Cys content was tripled to $10.5 \mathrm{~g} / \mathrm{kg}$. When indicated, drinking water through lixit was replaced by a bottle containing drinking water with $2 \%$ betaine hydrochloride (Bet; Sigma).

Table 1. Amino acid-defined diets used in the study.

\begin{tabular}{ccccccc}
\hline Component (g/kg) & $\begin{array}{c}\text { MET4.0 } \\
\text { (TD.170063) }\end{array}$ & $\begin{array}{c}\text { MET2.0 } \\
\text { (TD.170062) }\end{array}$ & $\begin{array}{c}\text { MET1.0 } \\
\text { (TD.170061) }\end{array}$ & $\begin{array}{c}\text { MET0.5 } \\
\text { (TD.110591) }\end{array}$ & $\begin{array}{c}\text { 3xCYS } \\
\text { (TD.170065) }\end{array}$ & $\begin{array}{c}\text { MET8.2 } \\
\text { (TD.01084) }\end{array}$ \\
\hline L-Alanine & 3.5 & 3.5 & 3.5 & 3.5 & 3.5 & 3.5 \\
L-Arginine.HCl & 12.1 & 12.1 & 12.1 & 12.1 & 12.1 & 12.1 \\
L-Asparagine & 6.0 & 6.0 & 6.0 & 6.0 & 6.0 & 6.0 \\
L-Aspartic Acid & 3.5 & 3.5 & 3.5 & 3.5 & 3.5 & 3.5 \\
L-Cystine & 3.5 & 3.5 & 3.5 & 3.5 & 10.5 & 3.5 \\
L-Glutamic Acid & 36.5 & 38.5 & 39.5 & 40.0 & 29.5 & 40.0 \\
Glycine & 23.04 & 23.04 & 23.04 & 23.04 & 23.04 & 23.3 \\
L-Histidine.HClxH ${ }_{2} \mathrm{O}$ & 4.5 & 4.5 & 4.5 & 4.5 & 4.5 & 4.5 \\
L-Isoleucine & 8.2 & 8.2 & 8.2 & 8.2 & 8.2 & 8.2 \\
L-Leucine & 11.1 & 11.1 & 11.1 & 11.1 & 11.1 & 11.1 \\
L-Lysine.HCl & 18.0 & 18.0 & 18.0 & 18.0 & 18.0 & 18.0 \\
L-Methionine & 4.0 & 2.0 & 1.0 & 0.5 & 4.0 & 8.2 \\
L-Phynylalanine & 7.5 & 7.5 & 7.5 & 7.5 & 7.5 & 7.5 \\
L-Proline & 3.5 & 3.5 & 3.5 & 3.5 & 3.5 & 3.5 \\
L-Serine & 3.5 & 3.5 & 3.5 & 3.5 & 3.5 & 3.5 \\
L-Threonine & 8.2 & 8.2 & 8.2 & 8.2 & 8.2 & 8.2 \\
L-Tryptophan & 1.8 & 1.8 & 1.8 & 1.8 & 1.8 & 1.8 \\
L-Tyrosine & 5.0 & 5.0 & 5.0 & 5.0 & 5.0 & 5.0 \\
\hline
\end{tabular}


Table 1. Cont.

\begin{tabular}{|c|c|c|c|c|c|c|}
\hline Component (g/kg) & $\begin{array}{c}\text { MET4.0 } \\
\text { (TD.170063) }\end{array}$ & $\begin{array}{c}\text { MET2.0 } \\
\text { (TD.170062) }\end{array}$ & $\begin{array}{c}\text { MET1.0 } \\
\text { (TD.170061) }\end{array}$ & $\begin{array}{c}\text { MET0.5 } \\
\text { (TD.110591) }\end{array}$ & $\begin{array}{c}3 \times C Y S \\
\text { (TD.170065) }\end{array}$ & $\begin{array}{c}\text { MET8.2 } \\
\text { (TD.01084) }\end{array}$ \\
\hline L-Valine & 8.2 & 8.2 & 8.2 & 8.2 & 8.2 & 8.2 \\
\hline Sucrose & 353.14 & 353.14 & 353.14 & 353.14 & 353.14 & 344.98 \\
\hline Corn Starch & 150.0 & 150.0 & 150.0 & 150.0 & 150.0 & 150.0 \\
\hline Maltodextrin & 150.0 & 150.0 & 150.0 & 150.0 & 150.0 & 150.0 \\
\hline Soybean Oil & 80.0 & 80.0 & 80.0 & 80.0 & 80.0 & 80.0 \\
\hline Cellulose & 30.0 & 30.0 & 30.0 & 30.0 & 30.0 & 30.0 \\
\hline $\begin{array}{c}\text { Mineral Mix } \\
(\text { AIN-93M-MX) }\end{array}$ & 35.0 & 35.0 & 35.0 & 35.0 & 35.0 & 35.0 \\
\hline $\mathrm{Ca}\left(\mathrm{H}_{2} \mathrm{PO}_{4}\right) 2 \times \mathrm{H}_{2} \mathrm{O}$ & 8.2 & 8.2 & 8.2 & 8.2 & 8.2 & 8.2 \\
\hline $\begin{array}{l}\text { Vitamin Mix } \\
\text { (AIN-93-VX) }\end{array}$ & 19.5 & 19.5 & 19.5 & 19.5 & 19.5 & 19.5 \\
\hline Choline bitartarate & 2.5 & 2.5 & 2.5 & 2.5 & 2.5 & 2.7 \\
\hline TBHQ (antioxidant) & 0.02 & 0.02 & 0.02 & 0.02 & 0.02 & 0.02 \\
\hline \multicolumn{7}{|c|}{ Summary Nutrient Information (\% by weight/\% kcal from) } \\
\hline Proteins & $14.9 / 15.1$ & $14.9 / 15.1$ & $14.9 / 15.1$ & $16.5 / 16.4$ & $15.0 / 15.2$ & $17.3 / 17.2$ \\
\hline Carbohydrates & $65.7 / 66.7$ & $65.7 / 66.7$ & $65.7 / 66.6$ & $65.7 / 65.6$ & $65.7 / 66.6$ & $64.9 / 64.8$ \\
\hline Fat & $8.0 / 18.3$ & $8.0 / 18.3$ & $8.0 / 18.3$ & $8.0 / 18.0$ & $8.0 / 18.3$ & $8.0 / 18.0$ \\
\hline $\begin{array}{l}\text { Energy density } \\
\text { (Kcal/g) }\end{array}$ & 3.9 & 3.9 & 3.9 & 4.0 & 3.9 & 4.0 \\
\hline
\end{tabular}

Met: Methionine.

\subsection{Animals and Study Design}

All procedures were performed at the University of Colorado Anschutz Medical Campus under the Institutional Animal Care and Use Committee (IACUC)-approved protocol\# 81, which complied with the Guide for the Care and Use of Laboratory Animals. The University is an Association for Assessment and Accreditation of Laboratory Animal Care (AAALAC)-accredited (\#00235), Public Health Service (PHS)-assured (\#D16-00171), and the United States Department of Agriculture (USDA)-licensed (\#84-R-0059) institution. "Human Only" (HO) mice were previously generated in our laboratory [9] and propagated and genotyped as described previously [10]. Both male and female young-adult HO mice (20-30 weeks of age) were used in two separate studies reported here. At the end of each study, mice were euthanized by $\mathrm{CO}_{2}$ asphyxiation followed by cervical dislocation.

The first study was designed to evaluate the efficacy of OT-58 treatment in the context of normal or reduced dietary Met intake without or with Bet or Cys supplementation. Figure 2 shows a graphical representation of the study design. Briefly, ten cohorts of $\mathrm{HO}$ mice $(\mathrm{n}=3$ males +3 females each) were transferred from the 2920X diet to amino acid-defined MET4.0/2.0/1.0/0.5 or 3xCYS diets. After a week of acclimation to new diets, drinking water in half of the cohorts was replaced with $2 \%$ Bet water ("+Bet" cohorts). After another week, all cohorts received seven doses of OT-58 $(8 \mathrm{mg} / \mathrm{kg}$ subcutaneously (SC)) once daily. Blood samples were collected on the following days: D1 (prior to the new diets), D7 (prior to the Bet supplementation), D12 (3 days before the first dose of OT-58), D16 and D22 (24 h after the first and last OT-58 dose, respectively).

The second study was designed to evaluate the efficacy of OT-58 treatment in the context of reduced, normal, and increased Met intake without or with Bet supplementation followed by fluctuations in Met intake. Figure 3 shows a graphical representation of the study design. Briefly, three cohorts of HO mice ( $n=4$ males +4 females each) were transferred from the 2920X diet to amino acid-defined MET4.0/8.2/0.5 diets. After two weeks of acclimation to new diets, drinking water for all cohorts was replaced with $2 \%$ Bet water. After three weeks of acclimation to the diets supplemented with Bet, all cohorts started to receive OT-58 ( $8 \mathrm{mg} / \mathrm{kg} \mathrm{SC}) 3 x$ a week (Monday-Wednesday-Friday) until the end of the study. After three weeks on diets, Bet supplementation and OT-58 injections, Bet was discontinued and replaced by regular drinking water through lixit. Two weeks after Bet withdrawal, 
fluctuations in Met intake were induced by switching between the three diets every two weeks as outlined in Figure 3. Blood samples were collected on the following days: D1 (prior to the new diets); D2 and D12 (acclimation to the new diets); D15, D24, and D33 (acclimation to Bet supplementation); D36, D45, and D54 (acclimation to OT-58 treatment); D57 and D68 (withdrawal of Bet); D71 and D82 (diet switch\#1); D85 and D96 (diet switch\#2); and lastly D99 and D110 (diet switch\#3).
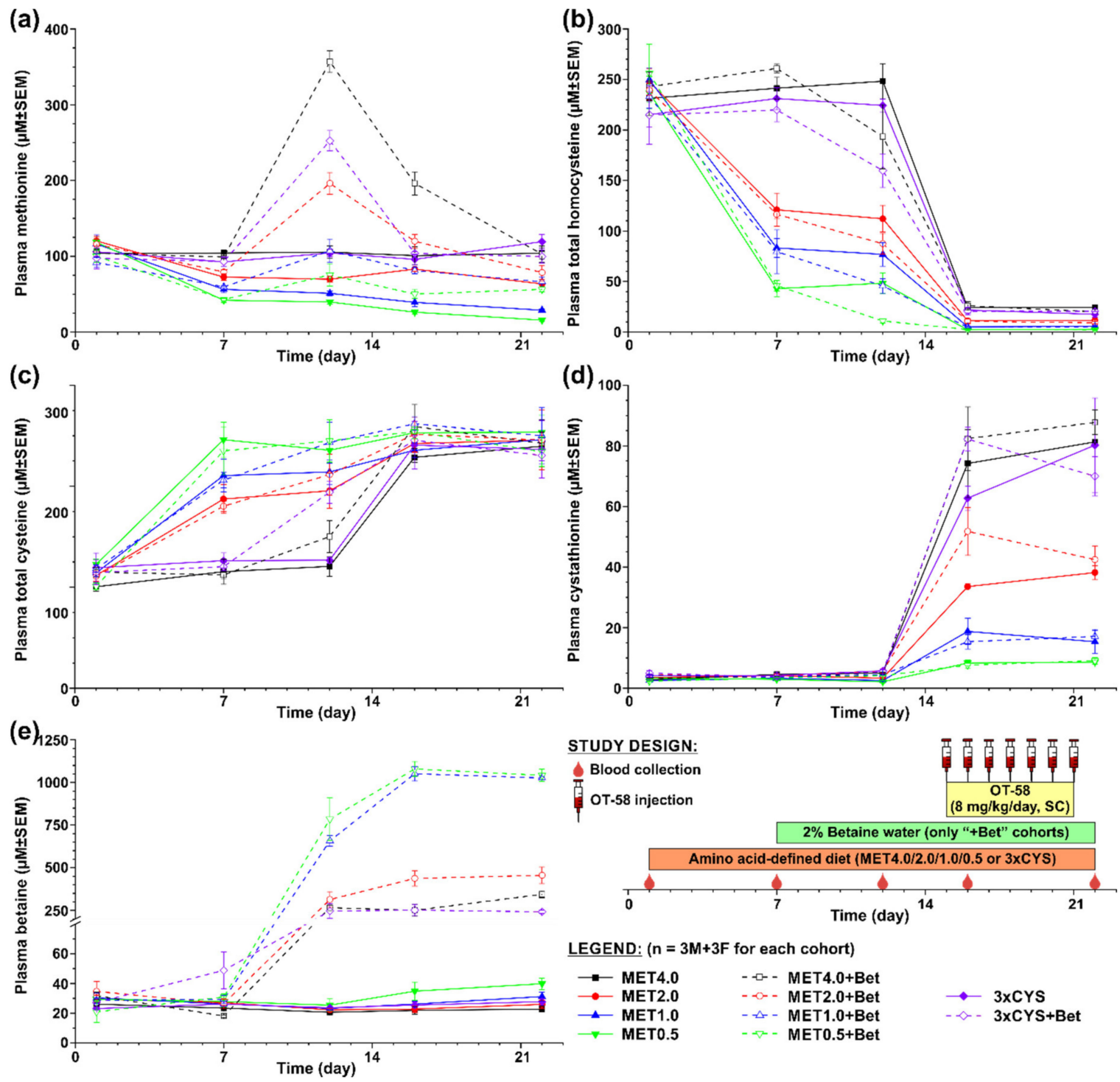

Figure 2. Short-term evaluation of OT-58 on the background of current standard of care for HCU. Ten HO mouse cohorts ( $\mathrm{n}=3$ males +3 females each) were set on a different dietary regime evaluating normal or decreased Met content in the diet (MET4.0/2.0/1.0/0.5), Bet supplementation (2\% Bet water), and dietary Cys supplementation ( $3 \times \mathrm{CYS}$ ). After acclimation, the efficacy of OT-58 treatment was assessed according to the study design shown in the bottom right corner. (a) Plasma Met, (b) plasma tHcy, (c) plasma tCys, (d) plasma Cth, (e) plasma Bet. Solid lines and closed symbols represent cohorts without Bet supplementation, while dashed lines and open symbols denote cohorts with Bet supplementation. Data points and error bars represent means and standard errors of means, respectively. 


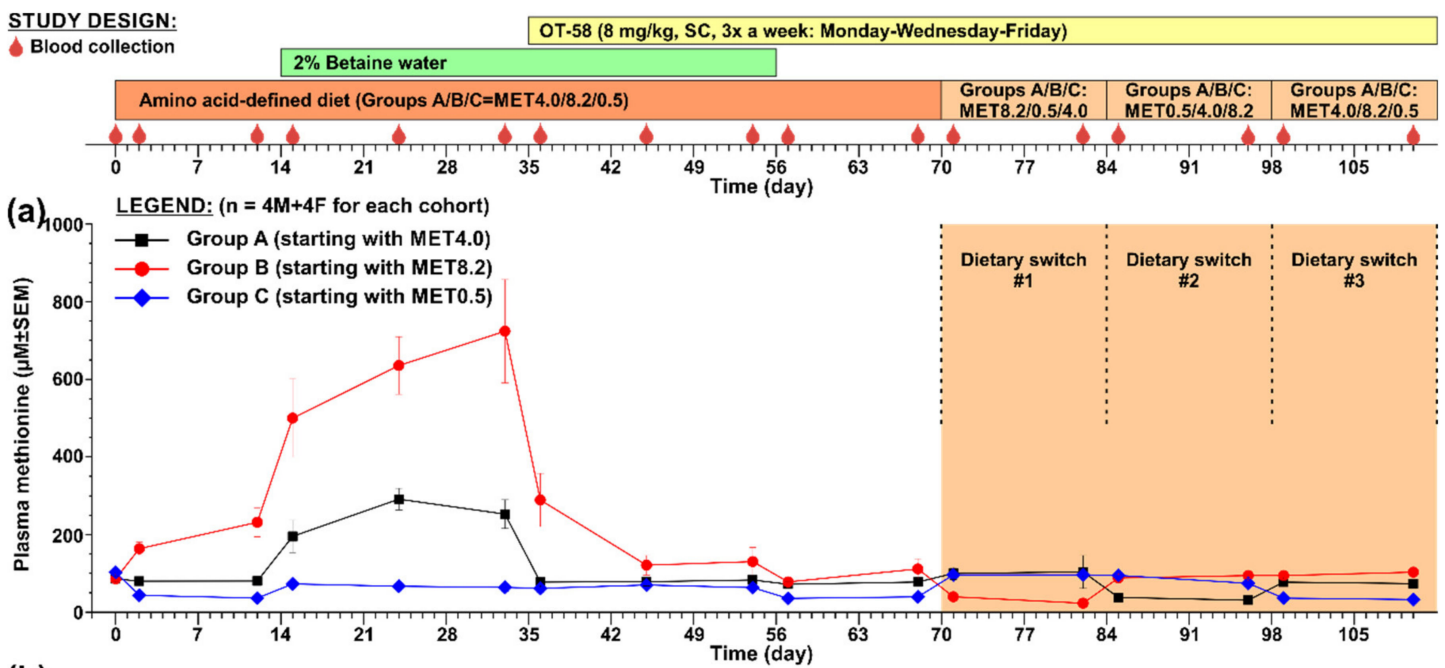

(b)
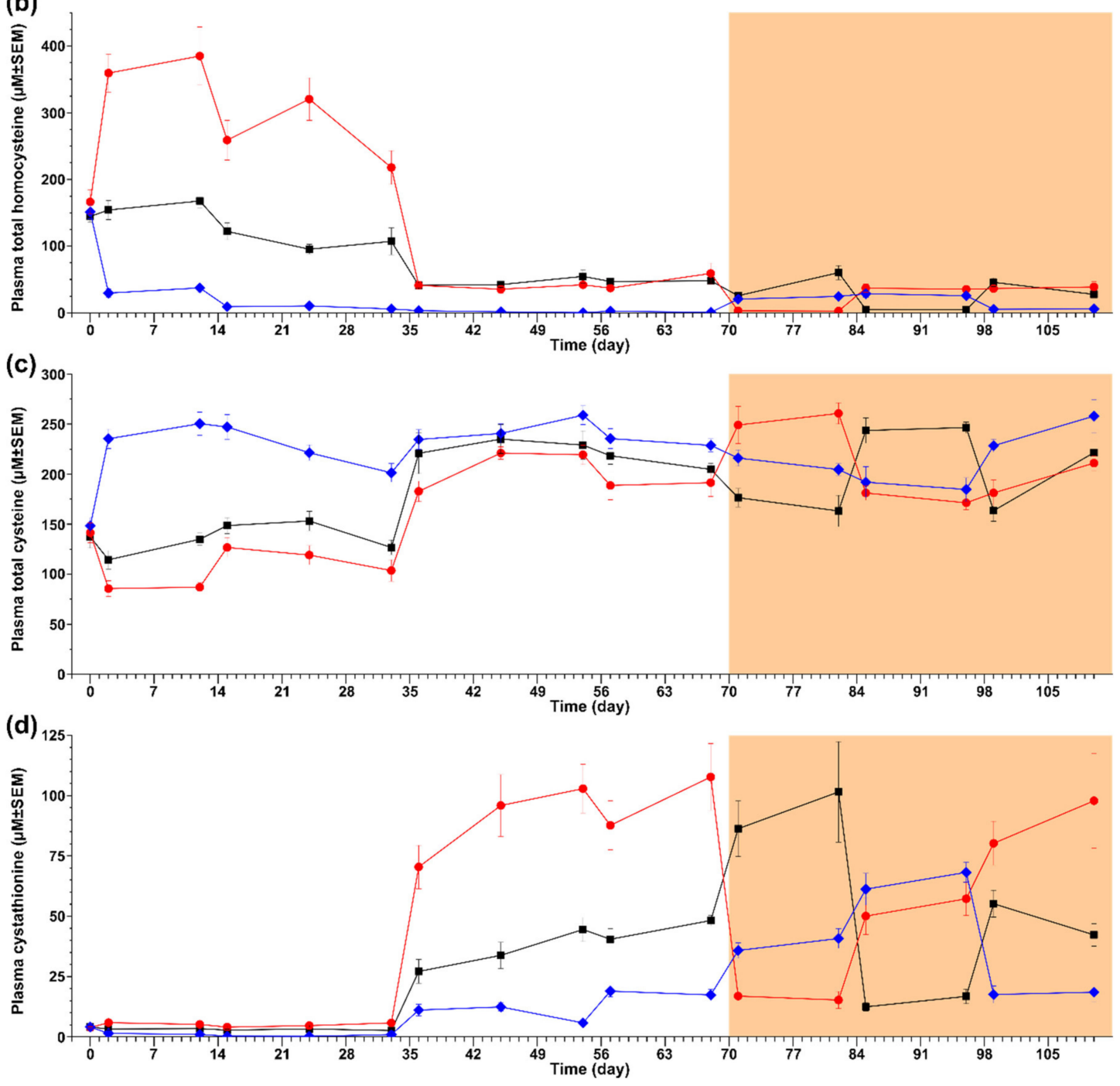

Figure 3. Long-term evaluation of OT-58. Three HO mouse cohorts ( $\mathrm{H}=4$ males +4 females each) were set on diets with reduced (MET0.5), normal (MET4.0), and increased (MET8.2) Met content later combined with Bet supplementation. OT-58 efficacy was evaluated on the background of these dietary regimes and later challenged by switching cohorts between the three diets as outlined in study design shown at the top. (a) Plasma Met, (b) plasma tHcy, (c) plasma tCys, (d) plasma Cth. Data points and error bars represent means and standard errors of means, respectively. 


\subsection{Blood Collection and Analysis}

Single-use lancets for submandibular bleedings were used for blood collections into BD Microtainer tubes with lithium heparin (Becto-Dickinson). The tubes were then centrifuged at 10,000 $\mathrm{g}$ for $5 \mathrm{~min}$, followed by the transfer of plasma into $1.5 \mathrm{~mL}$ tubes and storage at $-80^{\circ} \mathrm{C}$. Sulfur amino acid metabolites, namely Met, $\mathrm{tHcy}$, total cysteine ( $\mathrm{tCys}$ ), $\mathrm{Cth}$, and Bet, were determined by stable-isotope-dilution liquid chromatography tandem mass spectrometry (LC-MS/MS) as described elsewhere [11].

\subsection{Statistical Analysis}

All data are presented as mean \pm standard error of the mean (SEM). Statistical analyses were conducted by ANOVA followed by Tukey's multiple comparison test to determine significance, with values of $p<0.05$ being significant.

\section{Results}

\subsection{Short-Term Evaluation of OT-58 on the Background of Current Standard of Care for HCU}

In the first study, shown in Figure 2, we evaluated the impact of current standard of care for $\mathrm{HCU}$, i.e., dietary Met restriction often combined with Bet and/or Cys supplementation, on the plasma metabolic profile of $\mathrm{HO}$ mice in the absence and presence of OT-58. Five different diets were tested varying in content of Met (MET4.0/2.0/1.0/0.5) and Cys (3xCYS), with MET4.0 representing standard sulfur amino acid intake, MET2.0/1.0/0.5 representing graded Met restriction (50/25/12.5\% methionine content of MET4.0), and 3xCYS representing dietary supplementation of cysteine ( $3 \times$ more Cys compared to MET4.0 with the same Met content). Additionally, Bet supplementation was tested in conjunction with each of the diets ("+Bet" cohorts). Lastly, OT-58 (8 mg/kg/day, SC) was administered once daily for 7 days to all cohorts in order to study interaction of novel enzyme therapy with current standard of care in HO model of HCU.

Plasma Met decreased on average by 38\%, 48\%, and 63\% from the initial levels (107 $\pm 3 \mu \mathrm{M}$ on average) in mice fed with MET2.0, MET1.0, and MET0.5 diets, respectively $(p<0.05)$ but remained similar in mice on MET4.0 and 3xCYS (Figure 2a). Similarly, plasma tHcy declined on average by $52 \%, 67 \%$, and $81 \%$ from the initial levels ( $237 \pm 4 \mu \mathrm{M}$ on average) in mice fed with MET2.0, MET1.0, and MET0.5 diets, respectively $(p<0.01)$ but remained unchanged in mice on MET4.0 and 3xCYS (Figure 2b). Consequently, plasma tCys increased on average to $168 \%, 182 \%$, and $208 \%$ of the initial levels (113 $\pm 2 \mu \mathrm{M}$ on average) in mice on MET2.0, MET1.0, and MET0.5 diets, respectively $(p<0.05)$ but remained stable on MET4.0 and 3xCYS (Figure 2c). Plasma Cth and Bet remained largely unchanged during the acclimation period of mice to new diets $(3.7 \pm 0.3$ and $28.3 \pm 1.9 \mu \mathrm{M}$ on average, respectively; Figure 2d,e).

Bet supplementation resulted in a massive increase of plasma Bet from the initial levels on average 10-fold in mice on the MET4.0, MET2.0, and 3xCYS diets and 26-fold in mice on the MET1.0 and MET0.5 diets $(p<0.01$; Figure 2e). Elevated Bet resulted in plasma tHcy reduction by $\sim 26 \%$ from the initial levels in mice on the MET4.0 and 3xCYS diets $(p<0.05)$ and further reduced tHcy from the concentrations achieved by reduced Met intake by $25 \%, 43 \%$, and $76 \%$ in mice on the MET2.0, MET1.0, and MET0.5 diets, respectively ( $p<0.05$; Figure 2b). Consequently, plasma Met increased by Bet supplementation in correlation with Met intake and the initial plasma Met concentrations by 1.8-, 1.8-, and 2.5-times in mice on the MET0.5, MET1.0, and MET2.0, respectively, and 2.7- and 3.6-times in mice on the $3 \times$ CYS and MET4.0, respectively ( $p<0.05$; (Figure 2a). Bet supplementation also improved plasma tCys by $\sim 18 \%$ in mice on the MET2.0 and MET1.0 compared to that in those on diet alone and by $34 \%$ and $61 \%$ in mice on the MET4.0 and 3xCYS diets, respectively $(p<0.05$; Figure $2 c)$. Plasma Cth remained unchanged with Bet supplementation (Figure 2d).

Treatment with OT-58 normalized plasma tHcy in mice on the MET2.0/1.0/0.5 with or without Bet supplementation to 10,5 , and $2 \mu \mathrm{M}$, respectively, and substantially reduced it to 25 and $20 \mu \mathrm{M}$ in mice on the MET4.0 and 3xCYS with or without Bet supplementation, respectively $(p<0.01$; 
Figure $2 b)$. Plasma tCys was also normalized in all cohorts on average to $245 \mu \mathrm{M}$ representing 2.2-fold increase compared to the initial levels $(p<0.05$; Figure $2 c)$. As anticipated, OT-58 resulted in marked accumulation of plasma $\mathrm{Cth}$, which correlated with Met content in the diets but was not significantly affected by Bet supplementation. Specifically, plasma Cth increased compared to the initial levels on average 27-, 16-, 10-, 5-, and 3-fold in mice on MET4.0, 3xCYS, MET2.0, MET1.0, and MET0.5, respectively ( $p<0.01$; Figure 2d). Following OT-58 treatment, plasma Met decreased to levels similar to those induced by the diets alone. However, OT-58 resulted in an additional plasma Met drop by $40 \%$ and 50\% in mice the MET1.0 and MET0.5, respectively, compared to pre-OT-58 period $(p<0.05$; Figure 2a), which was completely prevented by Bet supplementation. Plasma Bet remained unchanged with OT-58 treatment (Figure 2e).

\subsection{Long-Term Evaluation of OT-58}

In the second study, shown in Figure 3, we evaluated the efficacy of OT-58 treatment to improve the plasma metabolic balance of HO mice in the context of reduced (MET0.5), normal (MET4.0), and increased (MET8.2) Met intake with or without Bet supplementation followed by fluctuations in Met intake to model for periods of non-compliance of HCU patients with the dietary regime.

Acclimation of $\mathrm{HO}$ mice to new diets resulted in a change of plasma Met concentrations in correlation with dietary Met intake. Specifically, plasma Met in mice on the MET4.0 remained unchanged, while it dropped by $65 \%$ in those on the MET0.5 and increased by $270 \%$ in those on the MET8.2 compared to the initial concentrations ( $p<0.01$; Figure 3a). Plasma tHcy followed a similar trend as that of Met, i.e., remained similar on the MET4.0, decreased by 75\% on the MET0.5, and increased by $232 \%$ on the MET8.2 compared to the initial concentrations ( $p<0.01$; Figure 3b). Conversely, plasma tCys levels were essentially normalized by the MET0.5 $(\sim 243 \mu \mathrm{M} ; p<0.01)$, remained unchanged on the MET4.0, and decreased by 38\% on the MET8.2 compared to the initial levels ( $p<0.05$; Figure 3c).

Bet supplementation exacerbated hypermethioninemia in $\mathrm{HO}$ mice on the MET8.2 additionally by up to 3.1-fold compared to the diet alone and increased plasma Met 3.1- and 1.7-fold in HO mice on the MET4.0 and MET0.5, respectively $(p<0.05$; Figure 3a). However, plasma tHcy was reduced by $36 \%, 43 \%$, and $85 \%$ compared to the diet alone in mice on the MET4.0, MET8.2, and MET0.5, respectively ( $p<0.05$; Figure $3 \mathrm{~b}$ ), with its concentration normalized in the cohort on the MET0.5 $(\sim 8 \mu \mathrm{M})$. Bet supplementation did not significantly change plasma tCys in HO mice on the MET4.0 and MET0.5 but improved it by $20 \%$ in those on the MET8.2 ( $p<0.05$; Figure 3c).

Treatment with OT-58 further decreased tHcy in mice on the MET0.5 diet to $\sim 2 \mu \mathrm{M}$ and substantially reduced hyperhomocysteinemia in $\mathrm{HO}$ mice on the MET4.0/8.2 to $\sim 43 \mu \mathrm{M}(p<0.01$; Figure 3b). Plasma Met remained unchanged in mice on the MET0.5 but was substantially decreased in those on the MET4.0 and MET8.2 to $\sim 80$ and $\sim 125 \mu \mathrm{M}$, respectively ( $p<0.05$; Figure 3a). On the other hand, plasma tCys increased and essentially normalized in all cohorts (MET0.5/4.0/8.2 $=\sim 250 / 234 / 220 \mu \mathrm{M}$, $p<0.05$; Figure 3c). Catalytic action of OT-58 resulted in marked accumulation of plasma Cth, which correlated with Met content in the diets. Specifically, plasma Cth increased compared to the initial levels on average 2.4-/8.6-/23.1-fold in mice on the MET0.5/4.0/8.2, respectively ( $p<0.01$; Figure 3d).

Withdrawal of Bet supplementation did not affect plasma tHcy levels in either cohort (Figure $3 b$ ). Plasma Met decreased on average by $41 \%, 10 \%$, and $27 \%$ in mice on the MET0.5/4.0/8.2, respectively $(p<0.05$; Figure 3a). Plasma tCys also decreased slightly by on average $10 \%, 8 \%$, and $13 \%$ in mice on the MET0.5/4.0/8.2, respectively ( $p<0.05$; Figure $3 c$ ) but remained within or close to normal levels (232/211/190 $\mu \mathrm{M}$ vs. $233 \mu \mathrm{M}$ in historical heterozygous HO controls [12]). Plasma Cth did not dramatically change after Bet withdrawal except in mice on the MET0.5, where it increased by $\sim 3.1$-fold compared to the value prior to the Bet withdrawal $(p<0.01$; Figure $3 \mathrm{~d})$.

In order to model for periods of non-compliance of HCU patients with their Met-restricted dietary regime, fluctuations of Met intake were achieved by three diet switch periods, each lasting for two weeks. Thus, each cohort received the MET0.5, MET4.0, and MET8.2 for two weeks and ended on 
the same diet as before the diet switch period (see Figure 3 for study design). The results could be summarized as follows. Plasma tHcy was maintained in the 20-60 $\mu \mathrm{M}$ range except for periods on the MET0.5, when it was normalized and fluctuated around $5 \mu \mathrm{M}$ (Figure $3 \mathrm{~b}$ ). Plasma tCys remained high and close to normal with the MET0.5 diet always contributing to full normalization (Figure 3c). Plasma Met remained stable and similar to the initial levels but always decreased on average by $63 \%$ when switched to the MET0.5 (Figure 3a). Lastly, plasma Cth remained markedly elevated compared to the initial levels due to OT-58 action on Hcy and correlated with the Met intake during each specific diet-switch period. Higher Met intake in the MET8.2 led to the highest plasma Cth concentrations (up to $101 \mu \mathrm{M}$ ), while lower Met intake in the MET0.5 led to the lowest plasma Cth concentrations (up o $18 \mu \mathrm{M}$; Figure 3d).

\section{Discussion}

Dietary management currently represents a primary therapeutic approach to pyridoxine non-responsive CBS-deficient HCU and an additional option for partially responsive HCU patients [3]. The first case report of successful dietary management of HCU with a diet low in Met and supplemented with cystine was published in 1966 by Komrower et al. [13] just a few years after HCU was first described in 1963 by Carson et al. [14]. Such dietary intervention resulted in a decreased influx of sulfur and, thus, lower production and accumulation of Hcy in affected individuals. As an elevated concentration of plasma Hcy has become a well-known risk factor for thromboembolic complications in HCU patients, Bet supplementation of either Met-restricted or unrestricted diet was introduced in early 1980s and showed further reduction or complete normalization of plasma Hcy levels $[15,16]$. This improvement, however, came at the price of elevation of plasma Met. Multiple studies have shown that loss of metabolic control is associated with serious complications, and, thus, HCU treatment must continue throughout the entire life [1,3,4,17]. However, compliance with Met restriction or Bet supplementation often deteriorates, particularly during adolescence, or is poor in general in individuals diagnosed later in life [4].

Due to the rarity of classical HCU and lack of appropriate animal models for many years, there has not been any development of new treatments for HCU until recently. To address unmet need and to provide new therapeutic options, we developed OT-58 and successfully evaluated it using various mouse models of HCU that are now available [5-7]. OT-58 performed well, correcting the plasma metabolic profile of HCU mice maintained on protein/Met unrestricted standard chow. In real-world scenarios, however, OT-58 efficacy will be confronted by the mainstay therapy (Met restriction, Cys and Bet supplementations) and lapses in compliance with the mainstay therapy (e.g., variable Met/protein intake, Bet withdrawal). Therefore, to gain further insight into the interplay of dietary management of HCU with OT-58, here we evaluated the short- and long-term efficacy of OT-58 on the background of increased, normal, and reduced Met intake with or without Cys or Bet supplementation. The effect of OT- 58 treatment on both the biochemical markers and the symptoms of the disease in mice was extensively studied in our lab using multiple mouse models of HCU [6-8]. The effect on biomarkers was similar in all mouse models. As it takes a longer time to see the impact of treatment on symptoms of HCU than the periods of various treatments included in this study, we decided to use HO mouse model. The HO mice recapitulate biochemical imbalance of HCU very well and do not suffer from poor survival or other adverse manifestations compared to other models available.

Met-restricted diets were effective in lowering of both plasma Met and tHcy, which decreased proportionally to the degree of Met restriction (Figure 2). However, even the most restrictive diet containing $12.5 \%$ of Met compared to control amino acid-defined MET4.0 diet (or 10\% compared to standard complete diet) did not achieve normalization. MET0.5 decreased initial tHcy from 237 and $151 \mu \mathrm{M}$ to 47 and $34 \mu \mathrm{M}$, respectively (Figures 2 and 3). In an I278T transgenic mouse model of HCU characterized by more severe phenotype and higher basal tHcy, severe Met restriction (i.e., $8.5-12.5 \%$ Met compared to standard diet) reduced tHcy from 357 and $423 \mu \mathrm{M}$ to 81 and $140 \mu \mathrm{M}$, respectively $[8,18]$. Together, the data suggest that the capacity of severe Met restriction to reduced tHcy depends on severity 
of the disease, level of CBS impairment, and, thus, initial tHcy concentration. Importantly, OT-58 normalized tHcy when combined with some level of Met restriction (MET2.0/1.0/0.5) and substantially reduced it when paired with normal or increased Met intake (MET4.0/8.2; Figures 2 and 3). These results indicate high efficacy of OT-58 to decrease and maintain tHcy below the recommended threshold of $100 \mu \mathrm{M}$ to prevent serious clinical complication in HCU patients [3]. Interestingly, a combination of OT-58 with MET1.0 and MET0.5 resulted in continuous decline of plasma Met (Figure 2). Low plasma Met is likely beneficial for HCU, as well as other indications, such as various cancers [19], but in case of $\mathrm{HCU}$, the level of Met restriction should still allow for normal growth and development [3]. Our data suggest that OT-58 in combination with moderate or no Met restriction markedly reduced plasma tHcy while it sustained normal plasma Met, which in turn should allow for normal growth and development. Accordingly, OT-58 may potentially offer a significant diet relaxation opportunity for human patients.

Cys supplementation on the background of normal Met intake failed to improve both plasma tHcy and tCys (Figure 2). This was unexpected, as previous study on I278T mice showed that $40 \mathrm{mM}$ $\mathrm{N}$-acetyl-L-cysteine, a more stable and water soluble Cys precursor, in drinking water increased tCys by $50 \%$ and reduced tHcy by $13 \%$ compared to levels in untreated I278T mice [20]. Either Cys supplementation (12.4 $\mathrm{mM}$ in drinking water) or taurine (160 $\mathrm{mM}$ in drinking water) normalized hepatic and blood taurine levels in $\mathrm{HO}$ mice, which lead to the normalization of HCU-induced regulatory changes in expression of the enzymes of Cys catabolic pathways [21]. However, no other improvements in phenotype of HCU mice were observed suggesting that low tCys may not be directly related to major clinical symptoms of HCU, thus, putting Cys supplementation in question. In fact, as the relationship between Cys and Hcy as thiol redox-active compounds is complex [22], data suggest that lowering of tHcy is more effective strategy to achieve tCys normalization compared to Cys supplementation. However, further research is clearly needed to clarify the role of Cys supplementation in HCU. The efficacy of OT-58 did not seem to be affected by 3-fold dietary Cys excess on the background of normal Met intake (Figure 2).

Bet supplementation should be considered as an adjunctive treatment in less compliant HCU patients who fail to achieve target levels of tHcy $[3,23]$. The use of Bet is generally safe, although its unpleasant taste and fishy odor often result in poor compliance [4]. In addition, extreme elevation of plasma Met during Bet treatment has been found to cause cerebral edemas, which resolve after Bet discontinuation [24,25]. Indeed, Bet supplementation resulted in plasma Met increase directly proportional to Met intake, while partially lowering tHcy (Figures 2 and 3). Interestingly, there was a marked variability in plasma betaine concentrations among mouse cohorts on different Met intake (Figure 2). While cohorts on Met4.0/2.0 showed 250-450 $\mathrm{MM}$ Bet, mice on the lower Met intake (MET1.0/0.5 diets) achieved plasma Bet concentrations of 650-1050 $\mu \mathrm{M}$. We hypothesize that the difference is related to intracellular availability of Hcy substrate for Bet-Hcy-methyltransferase (BHMT) and to the level of BHMT expression. Indeed, increased Met intake resulted in higher production of Hcy, which was available for BHMT-catalyzed re-methylation with Bet back to Met resulting in lower plasma Bet concentrations and increased plasma Met concentrations (Figure 2a,e). In addition, plasma tCys increased with Bet supplementation (Figure 2c). This effect can simply be explained by the fact that Hcy binds to Cys residues of plasma proteins (as well as free Cys) with a higher affinity than that of Cys thus replacing Cys, which results in plasma tCys decrease in HCU. Consequently, any treatment or intervention that reduces plasma tHcy prevents the loss of Cys and leads to the correction of plasma tCys. The efficacy of Bet in lowering tHcy diminished significantly over time in HO mice [26], which was later found to be alleviated by taurine treatment, which induced Bet-repressed expression of BHMT [27]. However, the long-term efficacy of Bet in HCU patients does not seem to be compromised [28]. Our studies showed that OT-58 provided additional benefit in correcting metabolic balance in $\mathrm{HO}$ mice over Bet supplementation alone and successfully competed with Bet for Hcy substrate. Regardless of Met restriction, OT-58 alone lowered tHcy to the same levels as achieved by co-administered Bet and OT-58 (Figure 2). Furthermore, OT-58 alone maintained plasma tHcy and improved metabolic control after Bet withdrawal irrespective of dietary Met intake (Figure 3). 
On the other hand, Bet supplementation maintained plasma Met on the background of severe Met restriction (12.5-25\% of normal intake), while co-administered OT-58 further decreased plasma Met in $\mathrm{HO}$ mice on MET1.0/0.5 diets (Figure 2). It is important to note the OT-58 does not replace the defective intracellular CBS. Whereas the native enzyme functions inside the cells, OT-58 does not enter the cells and operates in bloodstream, thus lowers intracellular Hcy indirectly. Betaine, on that other hand, serves as a methyl donor in the cytoplasm, and its therapeutic potential depends on the activity of intracellular BHMT to re-methylate Hcy to Met (Figure 1). Accordingly, OT-58 does not directly compete with betaine for Hcy inside the cells. In addition, Bet promotes the conservation of sulfur in the Met cycle. OT-58, on the other hand, processes Hcy irreversibly to Cth and, thus, removes the sulfur from the Met cycle (Figure 1). Together, our data suggest that Bet supplementation without severe Met restriction does not offer additional benefits when co-administered with OT-58. Moreover, exposure of HCU mice to OT-58 in the absence of Bet and without being primed with Bet supplementation was extensively studied by our group [6-8], showing Bet supplementation is not required to achieve good metabolic control on a background of normal Met intake combined with OT-58 treatment.

As OT-58 is currently tested in a phase I/II clinical trial (ClinicalTrials.gov ID NCT03406611), better understanding of OT-58 efficacy and its interaction with the current therapeutic options for HCU is important and highly relevant for study design, evaluation, and interpretation of the clinical trial results. Our previous data and those presented in this study clearly show that OT-58 retains its efficacy and performs well in a range of Met intakes in mouse models of HCU with different residual CBS activities [12]. Similarly, it is expected that these encouraging pre-clinical data may be translated to HCU patients, and, thus, OT-58 may become a valuable tool in the management and treatment of HCU. Specifically, OT-58 can efficiently reduce plasma tHcy below the $100 \mu \mathrm{M}$ threshold or normalize it entirely (Figures 2 and 3). Co-administration of Bet or Cys or their absence did not significantly affect OT-58's efficacy in improving metabolic balance (Figure 2). OT-58 maintained improved metabolic balance during periods of highly variable dietary Met intake (Figures 2 and 3). Lastly, our previous long-term studies using different mouse models of HCU showed no reduction of OT-58 efficacy over time, indicating no significant immune response to OT-58 in mice [6-8], although the possibility that OT-58 will induce neutralizing antibodies in some HCU patients cannot be ruled out. Taken together, this pre-clinical study on HO mouse model of HCU suggests that OT-58 may represent a novel, highly effective, enzyme therapy for HCU performing optimally in the presence or absence of previous treatments, such as Met/protein restriction or Cys/Bet supplementation and during periods of poor compliance of HCU patients with dietary management of the disease. It remains to be seen how OT-58 will perform in the real world, but our study gives an optimistic outlook for this and similar enzyme therapies in development for HCU [29].

Author Contributions: Conceptualization, T.M.; methodology, T.M.; formal analysis, T.M.; investigation, I.P., T.M.; resources, E.M.B. and F.G.; writing—original draft preparation, T.M.; writing—review and editing, T.M. and E.M.B.; visualization, T.M.; supervision, T.M.; project administration, T.M.; funding acquisition, T.M. All authors have read and agreed to the published version of the manuscript.

Funding: This study was supported by a research grant from Orphan Technologies Ltd. (AWD-111186 and AWD-182790 to T.M.). T.M. also acknowledges financial support from the American Heart Association Scientist Development Grant (16SDG30040000) and the HCU Network North America and Australia Research grant.

Acknowledgments: We acknowledge the life-long accomplishments of Jan P. Kraus in the field of inborn errors of metabolism, particularly CBS biochemistry and homocystinuria. Kraus initiated the work on OT-58, but unfortunately passed away last year. Therefore, we dedicate this article to his memory. The authors would also like to acknowledge Carla Ray, Linda Farb, Sally Stabler, Robert Allen, Erland Arning, and Teodoro Bottiglieri for the determination of plasma sulfur amino acid metabolites.

Conflicts of Interest: The research was primarily funded by Orphan Technologies Ltd., a private pharmaceutical company developing OT-58 enzyme replacement therapy for classical HCU. T.M. provided ad hoc consulting services to Orphan Technologies Ltd. E.M.B. and F.G. are employees of Orphan Technologies Ltd. T.M. and E.M.B. are inventors on patents related to the processes and products referred here (U.S. patents 9,034,318 and 9,243,239). 


\section{References}

1. Mudd, S.H.; Levy, H.L.; Kraus, J.P. Disorders of Transsulfuration. In The Metabolic and Molecular Bases of Inherited Disease, 8th ed.; Scriver, C.R., Beaudet, A.L., Sly, W.S., Valle, D., Childs, B., Kinzler, K., Vogelstein, B., Eds.; McGraw-Hill: New York, NY, USA, 2001; pp. 2007-2056.

2. Zuhra, K.; Augsburger, F.; Majtan, T.; Szabo, C. Cystathionine-beta-Synthase: Molecular Regulation and Pharmacological Inhibition. Biomolecules 2020, 10, 697. [CrossRef]

3. Morris, A.A.; Kozich, V.; Santra, S.; Andria, G.; Ben-Omran, T.I.; Chakrapani, A.B.; Crushell, E.; Henderson, M.J.; Hochuli, M.; Huemer, M.; et al. Guidelines for the diagnosis and management of cystathionine beta-synthase deficiency. J. Inherit. Metab. Dis. 2017, 40, 49-74. [CrossRef]

4. Walter, J.H.; Wraith, J.E.; White, F.J.; Bridge, C.; Till, J. Strategies for the treatment of cystathionine $\beta$-synthase deficiency: The experience of the Willink Biochemical Genetics Unit over the past 30 years. Eur. J. Pediatrics 1998, 157 (Suppl. 2), S71-S76. [CrossRef]

5. Majtan, T.; Park, I.; Carrillo, R.S.; Bublil, E.M.; Kraus, J.P. Engineering and Characterization of an Enzyme Replacement Therapy for Classical Homocystinuria. Biomacromolecules 2017, 18, 1747-1761. [CrossRef]

6. Majtan, T.; Hulkova, H.; Park, I.; Krijt, J.; Kozich, V.; Bublil, E.M.; Kraus, J.P. Enzyme replacement prevents neonatal death, liver damage, and osteoporosis in murine homocystinuria. FASEB J. 2017, 31, 5495-5506. [CrossRef]

7. Majtan, T.; Jones, W., Jr.; Krijt, J.; Park, I.; Kruger, W.D.; Kozich, V.; Bassnett, S.; Bublil, E.M.; Kraus, J.P. Enzyme Replacement Therapy Ameliorates Multiple Symptoms of Murine Homocystinuria. Mol. Ther. 2018, 26, 834-844. [CrossRef]

8. Majtan, T.; Park, I.; Cox, A.; Branchford, B.R.; di Paola, J.; Bublil, E.M.; Kraus, J.P. Behavior, body composition, and vascular phenotype of homocystinuric mice on methionine-restricted diet or enzyme replacement therapy. FASEB J. 2019, 33, 12477-12486. [CrossRef]

9. Maclean, K.N.; Sikora, J.; Kozich, V.; Jiang, H.; Greiner, L.S.; Kraus, E.; Krijt, J.; Overdier, K.H.; Collard, R.; Brodsky, G.L.; et al. A novel transgenic mouse model of CBS-deficient homocystinuria does not incur hepatic steatosis or fibrosis and exhibits a hypercoagulative phenotype that is ameliorated by betaine treatment. Mol. Genet. Metab. 2010, 101, 153-162. [CrossRef] [PubMed]

10. Bublil, E.M.; Majtan, T.; Park, I.; Carrillo, R.S.; Hulkova, H.; Krijt, J.; Kozich, V.; Kraus, J.P. Enzyme replacement with PEGylated cystathionine beta-synthase ameliorates homocystinuria in murine model. J. Clin. Investig. 2016, 126, 2372-2384. [CrossRef] [PubMed]

11. Arning, E.; Bottiglieri, T. Quantitation of S-Adenosylmethionine and S-Adenosylhomocysteine in Plasma Using Liquid Chromatography-Electrospray Tandem Mass Spectrometry. Methods Mol. Biol. 2016, 1378, 255-262. [CrossRef]

12. Majtan, T.; Park, I.; Bublil, E.M.; Kraus, J.P. Enzyme replacement therapy prevents loss of bone and fat mass in murine homocystinuria. Hum. Mutat. 2018, 39, 210-218. [CrossRef] [PubMed]

13. Komrower, G.M.; Lambert, A.M.; Cusworth, D.C.; Westall, R.G. Dietary treatment of homocystinuria. Arch. Dis. Child. 1966, 41, 666-671. [CrossRef]

14. Carson, N.A.J.; Cusworth, D.C.; Dent, C.E.; Field, C.M.B.; Neill, D.W.; Westall, R.G. Homocystinuria: A new inborn error of metabolism associated with mental deficiency. Arch. Dis. Child. 1963, 38, 425-436. [CrossRef]

15. Smolin, L.A.; Benevenga, N.J.; Berlow, S. The use of betaine for the treatment of homocystinuria. J. Pediatrics 1981, 99, 467-472. [CrossRef]

16. Wilcken, D.E.L.; Wilcken, B.; Dudman, N.P.B.; Tyrrell, P.A. Homocystinuria-The effects of betaine in the treatment of patients not responsive to pyridoxine. N. Engl. J. Med. 1983, 309, 448-453. [CrossRef]

17. Yap, S.; Naughten, E. Homocystinuria due to cystathionine beta-synthase deficiency in Ireland-25 years experience of a newborn screened and treated population with reference to clinical outcome and biochemical control. J. Inherit. Metab. Dis. 1998, 21, 738-747. [CrossRef]

18. Gupta, S.; Wang, L.; Kruger, W.D. Betaine supplementation is less effective than methionine restriction in correcting phenotypes of CBS deficient mice. J. Inherit. Metab. Dis. 2016, 39, 39-46. [CrossRef]

19. Hoffman, R.M. Development of recombinant methioninase to target the general cancer-specific metabolic defect of methionine dependence: A 40-year odyssey. Expert Opin. Biol. Ther. 2015, 15, 21-31. [CrossRef]

20. Gupta, S.; Kruger, W.D. Cystathionine beta-synthase deficiency causes fat loss in mice. PLoS ONE 2011, 6, e27598. [CrossRef] 
21. Jiang, H.; Stabler, S.P.; Allen, R.H.; Abman, S.H.; Maclean, K.N. Altered hepatic sulfur metabolism in cystathionine beta-synthase-deficient homocystinuria: Regulatory role of taurine on competing cysteine oxidation pathways. FASEB J. 2014, 28, 4044-4054. [CrossRef]

22. Lee, P.J.; Briddon, A. A rationale for cystine supplementation in severe homocystinuria. J. Inherit. Metab. Dis. 2007, 30, 35-38. [CrossRef]

23. Lawson-Yuen, A.; Levy, H.L. The use of betaine in the treatment of elevated homocysteine. Mol. Genet. Metab. 2006, 88, 201-207. [CrossRef]

24. Yaghmai, R.; Kashani, A.H.; Geraghty, M.T.; Okoh, J.; Pomper, M.; Tangerman, A.; Wagner, C.; Stabler, S.P.; Allen, R.H.; Mudd, S.H.; et al. Progressive cerebral edema associated with high methionine levels and betaine therapy in a patient with cystathionine beta-synthase (CBS) deficiency. Am. J. Med. Genet. 2002, 108, 57-63. [CrossRef]

25. Devlin, A.M.; Hajipour, L.; Gholkar, A.; Fernandes, H.; Ramesh, V.; Morris, A.A. Cerebral edema associated with betaine treatment in classical homocystinuria. J. Pediatrics 2004, 144, 545-548. [CrossRef]

26. Maclean, K.N.; Jiang, H.; Greiner, L.S.; Allen, R.H.; Stabler, S.P. Long-term betaine therapy in a murine model of cystathionine beta-synthase deficient homocystinuria: Decreased efficacy over time reveals a significant threshold effect between elevated homocysteine and thrombotic risk. Mol. Genet Metab. 2012, 105, 395-403. [CrossRef]

27. Maclean, K.N.; Jiang, H.; Phinney, W.N.; Keating, A.K.; Hurt, K.J.; Stabler, S.P. Taurine alleviates repression of betaine-homocysteine S-methyltransferase and significantly improves the efficacy of long-term betaine treatment in a mouse model of cystathionine beta-synthase-deficient homocystinuria. FASEB J. 2019, 33, 6339-6353. [CrossRef]

28. Valayannopoulos, V.; Schiff, M.; Guffon, N.; Nadjar, Y.; Garcia-Cazorla, A.; Martinez-Pardo Casanova, M.; Cano, A.; Couce, M.L.; Dalmau, J.; Pena-Quintana, L.; et al. Betaine anhydrous in homocystinuria: Results from the RoCH registry. Orphanet J. Rare Dis. 2019, 14, 66. [CrossRef]

29. Bublil, E.M.; Majtan, T. Classical homocystinuria: From cystathionine beta-synthase deficiency to novel enzyme therapies. Biochimie 2020, 173, 48-56. [CrossRef]

(C) 2020 by the authors. Licensee MDPI, Basel, Switzerland. This article is an open access article distributed under the terms and conditions of the Creative Commons Attribution (CC BY) license (http://creativecommons.org/licenses/by/4.0/). 